\title{
Políticas Públicas en América Latina: abordajes a partir de Amartya Sen
}

\author{
Henrique Kujawa ${ }^{1}$ \\ Neuro José Zambam²
}

\section{Contribución en la Sección Panorama Social}

Resumen: Este trabajo tiene como objetivo general fundamentar la comprensión de políticas públicas para demostrar su repercusión, sea simbólica, sea en el cotidiano personal, familiar y social como forma de efectivizar la justicia social a través del ejercicio de la libertad, participación social en el poder de decisión y de la mejora de las capacidades (capabilities). La construcción del artículo surgió a partir de la revisión bibliográfica y de entrevistas realizadas con beneficiarios de las políticas públicas analizadas. Del impulso de políticas públicas estructuradas y eficaces, a partir de decisiones políticas adecuadas, dependen la superación de las graves desigualdades, la búsqueda de la equidad social y la renovación de la democracia.

\section{Introducción}

Las desigualdades sociales, además de ser un mal persistente en América Latina y en otras partes del mundo, han aumentado significativamente en las últimas décadas. Los avances tecnológicos, el proceso de globalización, el aumento de producción y circulación de bienes no han garantizado una distribución equitativa de los resultados, ni tampoco la garantía de las condiciones básicas de vida para una parcela significativa de personas. El hambre, la mortalidad infantil, la falta de acceso a educación y a los servicios básicos de salud continúan siendo grandes obstáculos para una vida satisfactoria de los individuos y grupos sociales de la región.

Desde la perspectiva de Sen, no es posible pensar el desarrollo y la ampliación de justicia, sin buscar soluciones para estas cuestiones que restringen las posibilidades de que los individuos desplieguen sus capacidades (capabilities) para ejercer la libertad de elección. Capacidades que consideran relevantes para sí y para las personas cercanas. La ampliación de las libertades instrumentales, entre las que se encuentran la seguridad protectora, es fundamental para garantizar el desarrollo y para eliminar las injusticias evitables.

\footnotetext{
${ }^{1}$ Doctor en Ciencias Sociales (UNISINOS). Correo electrónico: henrique.kujawa@imed.edu.br

2 Pos-doctor en Filosofía en la Universidad del Valle del Rio de los Sinos (UNISINOS). Doctor en Filosofía por la PUCRS. Correo electrónico: neuro.zambam@imed.edu.br
} 
No se trata de volvernos contra los procesos de globalización o abdicar del dinamismo del mercado en el avance tecnológico, o de la producción y circulación de mercaderías, pero sí discutimos la división equitativa de los beneficios generados por la humanidad (Sen y Kliksberg, 2010). Las políticas públicas poseen un papel primordial para que eso ocurra, garantizando el derecho a la alimentación, educación, servicios de salud, habitación y seguridad.

Es en esta perspectiva que gana importancia la reflexión sobre cómo pensar políticas públicas como mecanismo de desarrollo y de promoción de justicia. Proponemos una reflexión que apunta a la contribución de Sen sobre la construcción de políticas públicas a partir de los presupuestos de desarrollo y de justicia. El objetivo general de este texto es analizar las contribuciones de la teoría de justicia y de la concepción del desarrollo de Sen para formular, monitorear y evaluar políticas públicas. Para ello, se propone un rescate de la concepción de desarrollo, de justicia y de políticas públicas para Sen; construiremos referencias que puedan indicar una matriz para analizar la formulación e implantación de políticas públicas; y haremos un ejercicio de análisis de políticas públicas específicas a partir de esta prerrogativa.

Las políticas públicas analizadas serán el Proyecto Canaán, desenvuelto en el ámbito del programa Mi Casa Mi Vida (MCMV), que tiene como objetivo ampliar el derecho a la vivienda en Brasil. Y, la segunda, es el Programa Apoyar y Comprometer (PAC) que tiene como objetivo colocar profesionalmente a personas con alto grado de vulnerabilidad social, para que gradualmente, no dependan más de servicios de asistencia social. Los dos proyectos fueron implementados en la ciudad de Passo Fundo, Rio Grande do Sul, Brasil.

\section{El abordaje de Sen sobre las políticas públicas}

La estrategia que procura efectuar el desarrollo humano, social económico y sostenible o, en la visión de Sen, como promoción y expansión de las libertades de las personas, tiene en las políticas públicas un mecanismo privilegiado para su realización por que se constituye como "[...] un importante recurso que los Estados disponen para enfrentar los graves dilemas sociales que amenazan su funcionamiento y su organización visando justicia social" (Zambam y Kujawa, 2017, p. 62). Este es un mecanismo por medio del cual los gobiernos buscan ecualizar, especialmente, las desigualdades sociales.

Las políticas públicas precisan contemplar las diferencias, los intereses y modelos que están en la sociedad, específicamente entre el capital humano y el crecimiento económico. Sen (2000, p. 334) es enfático sobre el significado de eso para la estructura de las políticas públicas, pues estas precisan contribuir para el desarrollo social y político. 
Ampliar el abordaje, más allá del desarrollismo, implica comprender a las personas como el objetivo más importante del proceso de organización social, crecimiento económico y equidad social. En la misma perspectiva, Sen (1999) es implacable en la evaluación del distanciamiento entre ética y economía en la modernidad y sus nefastas consecuencias para la concepción de persona.

La calidad de las políticas públicas está íntimamente relacionada con la vitalidad de la democracia por ser un sistema en el que prima la participación, la superación de las desigualdades, el ejercicio de la tolerancia y la promoción de la libertad. El vigor de las políticas públicas depende de su capacidad de disminuir las grandes desigualdades, de promover las condiciones electorales y de las capacidades (capabilities), así como también de ampliar la participación y corregir las distorsiones en el campo de la salud, la educación y la seguridad protectora.

La relevancia de la superación de las situaciones sociales degradantes -como el analfabetismo, las enfermedades evitables y la violencia- con políticas públicas eficaces es subrayada por Sen (2001) como condición para la libertad de las personas y la estabilidad social. El debate académico sobre los objetivos, la formulación, la implantación y la evaluación de las políticas públicas ha recibido, desde mediados del siglo XX, significativa atención de investigadores. Diferentes modelos teóricos y operacionales discuten la forma con que determinados temas entran en la agenda y crean la necesidad de elaboración de políticas públicas, cuál es el papel del Estado y de las instituciones, de qué forma la sociedad y los individuos pueden o tienen poder de ejercer influencias sobre estas agendas, la relación entre el Estado y la sociedad organizada, etc.

En esta perspectiva, resultan centrales la construcción de una agenda, la formulación de políticas públicas; su implementación y su evaluación, por cuanto ella tiene condiciones de contribuir para el desarrollo, o sea "su valor precisa depender del impacto que (...) tienen en la vida y libertades de las personas envueltas, que necesita ser central para la idea de desarrollo" (Sen, 2011, p. 380).

La calidad de las políticas públicas está íntimamente relacionada con la vitalidad de la democracia por ser un sistema que prima por la participación, superación de las desigualdades, ejercicio de la tolerancia y promoción de la libertad. La democracia, en tanto, es la condición política necesaria y más eficaz para promover el desarrollo y la justicia. Como demuestra el grafico que se presenta a continuación, realizado por los autores: 


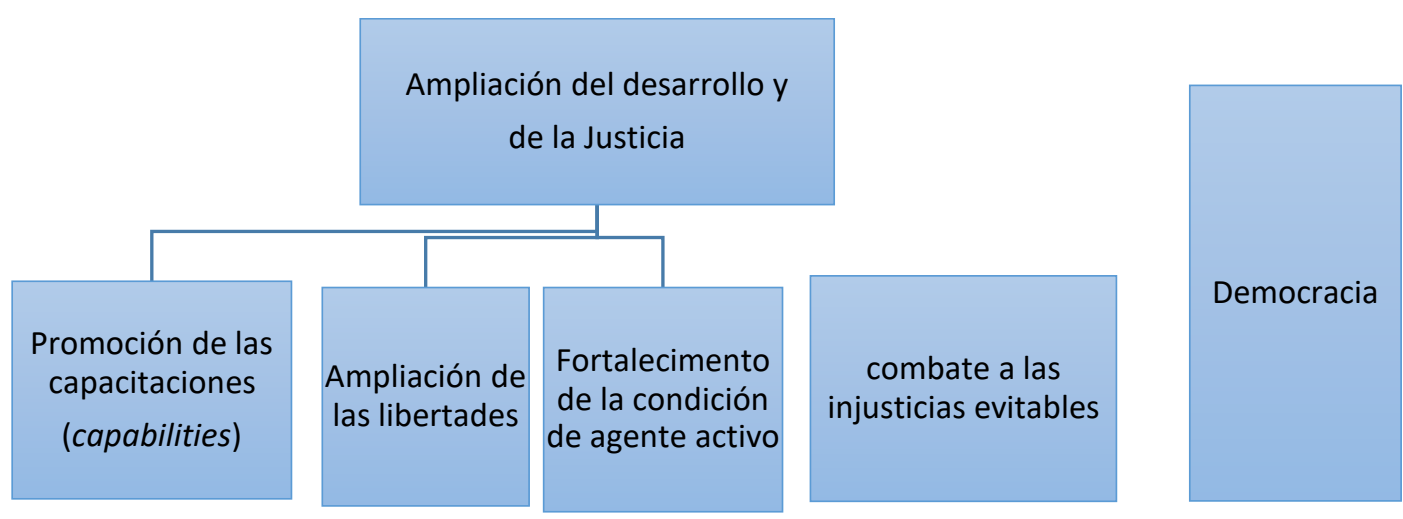

En relación a la democracia, Sen (2000, p. 180), apunta que "los derechos políticos y civiles especialmente los relacionados a la garantía de discusión, debate, crítica y discordancias abiertas son centrales para el proceso de generación de decisiones bien fundamentadas y reflexionadas". En la misma perspectiva, reflexionando sobre la importancia del ejercicio de la democracia, Nussbaum (2015, p. 77) apunta que: "las democracias del mundo entero están subestimando y, por lo tanto, ignorando competencias de las que precisamos desesperadamente para mantener las democracias vivas, respetuosas y responsables".

Otra cuestión fundamental es la relación de las responsabilidades del Estado y, por otro lado, del mercado y de los individuos. Sen, en el transcurso de su obra, apunta que tanto el Estado como el mercado tienen un papel importante en la promoción del desarrollo y de la justicia.

\section{Proyectos Canaan y Programa Apoyar y Comprometer: análisis a partir de presupuestos de criterios desde Sen}

Se afirmó que el análisis de las políticas públicas a partir de las perspectivas de Sen requiere que tengamos como presupuesto la ampliación del desenvolvimiento y de la justicia y como criterio la promoción de capacidades (capabilities), de las libertades, de la condición de agente y la capacidad de eliminar injusticias evitables. Haremos un ejercicio para demostrar las condiciones de aplicación de esta matriz para analizar dos políticas públicas en Passo Fundo ${ }^{3}$, Rio Grande do Sul, Brasil.

La elección del Proyecto Canaán y del Programa Apoyar y Comprometer ocurre a partir de tres motivos. El primero es el hecho de que estas políticas apuntan al acceso a la vivienda y al amparo de personas en situación de alta vulnerabilidad, dos cuestiones básicas que hacen parte de lo que Sen denomina libertad instrumental de "seguridad protectora" (Sen,

${ }^{3}$ La ciudad de Passo Fundo poseía, según el censo de 2010, 184.826 habitantes, y la estimativa para 2018 era de 201.767 habitantes (IBGE, 2018). 
2000, p. 54). El segundo motivo es que, por estas políticas públicas son consideradas como ejemplares, diferenciándose inclusive de las políticas tradicionales que centran sus objetivos en la atención de aspectos económicos, materiales y de bienestar. Por fin, dado que la aplicación de la matriz propuesta, coherente con la concepción de Sen, no puede resumirse a estadísticas, puesto que es necesario oír a los sujetos beneficiarios para identificar en qué medida se logró la eliminación de injusticias evitables, la ampliación de capacitaciones, de las libertades, de la condición de agente y de la democracia. Esta experiencia de indagación contó con la realización de siete entrevistas (semiestructuradas) en el Canaán y seis en el PAC, en el marco de la organización de grupos focales y observación in loco.

\subsection{Proyecto Canaán}

Vale decir que la urbanización brasileña, principalmente después de mediados del siglo XX, también es marcada por la especulación inmobiliaria y la adecuación de los espacios a la producción capitalista (Maricato, 2014; Rolnick, 2015). Descorre de eso una urbanización desordenada, un crecimiento exponencial de las regiones periféricas, la precarización y la falta de vivienda. Datos de la Fundación Pinheiro (2017), demuestran que, en Brasil en el año de 2015, ese déficit representaba 6.186.503 de domicilios.

En 2009, en medio a la crisis inmobiliaria de los EE.UU. y de la amenaza de estanflación económica, el Gobierno brasileño creó el programa Mi Casa Mi Vida (MCMV) que unificó programas anteriores objetivando una agilidad mayor en la construcción de viviendas de interés social para disminuir el déficit y también acelerar la economía de la construcción civil y la generación de nuevos puestos de trabajo.

Una de las modalidades del referido programa fue Mi Casa Mi Vida - Entidades (MCMV-E) destinado a financiar viviendas sociales para la población de baja renta, con recursos subsidiados por el Tesoro Nacional. En esta modalidad, los valores son repasados por la Caixa Económica Federal para una Entidad Organizadora que puede ser pública o privada sin fines lucrativos, que se vuelve responsable por planear, constituir el grupo de beneficiarios y construir las casas. Todo el proceso es fiscalizado por la CEF.

El Canaán fue un proyecto construido por el Grupo de Mujeres Unidos Venceremos en el Barrio Leonardo Ilha. El Grupo de mujeres surgió en 1999 con el objetivo de organizar a las mujeres del barrio, teniendo como primera acción la construcción de una guardería comunitaria para atender hijos, aumentando la empleabilidad de las madres. Posteriormente, el Grupo de Mujeres actuó con programas de alfabetización y ampliación de la escolaridad, así como también desde la oferta de cursos de formación profesional (Kujawa y Zambam, 2018). 
En 2006 la entidad tuvo la primera experiencia con la construcción y reforma de casas a través del Programa de Crédito Solidario, y en 2010, creó el proyecto Canaán con el que construyó 210 viviendas. Las obras iniciaron en 2013 y fueron concluidas en 2018.

\subsection{Programa Apoyar y Comprender (PAC)}

EI PAC tuvo origen en 2003 cuando el poder público municipal de Passo Fundo creó un registro de desempleados en situación de vulnerabilidad social con el fin de colocarlos en tareas, principalmente, en la limpieza de la ciudad, a cambio de remuneración mensual. A partir de 2008, pasó a exigirse el aumento de la escolaridad o la realización de cursos de formación profesional en contrapartida de los beneficiarios. En 2013 el PAC se transformó en una política pública reglamentada por la Ley 5009/2013.

Los recursos para el PAC provienen del presupuesto municipal, siendo que los beneficiarios reciben $80 \%$ del salario mínimo en dinero, y $20 \%$ en alimentos, más dos vales para transportes (pasajes de ómnibus) por día de trabajo. El número medio de personas vinculadas al programa en los últimos cinco años es de 150 por año, aunque el límite del presupuesto impide que se atienda a todos los que requerían el Programa; hoy la lista de espera es de más de 1000 personas, y puede ser considerada bastante expresiva si se compara con la capacidad de inclusión (150/año) de beneficiarios/as (Kujawa y Zamban, 2018).

\section{Análisis de las dos políticas públicas a partir de los presupuestos de Desarrollo y de Justicia}

Tratándose de la realidad urbana, tanto el déficit de viviendas como el desempleo y la falta de renta son injusticias evitables o la falta de garantía de seguridad protectora, perjudican el desarrollo, limitando las capacidades (capabilities), reduciendo las libertades de agente activo de los individuos y de las familias. En relación al Canaán, todos los beneficiarios no tenían casa propia y la gran mayoría pagaba alquiler. Cuando se preguntó acerca de lo que representó para la vida de los/as beneficiarios/as que debían pagar alquiler, la respuesta más frecuente fue "más comida en la mesa", o "el alquiler tomaba la mayor parte de nuestro dinero", "ahora puedo hasta comprar un yogurt, una fruta, un chocolate para mis hijos". En este mismo sentido, una madre de familia respondió que suspender el pago del alquiler significa:

ropas mejores, calzados mejores, viajar, yo no conseguía salir de Passo Fundo, ahora conseguimos. Ahora estoy planeando un viaje de vacaciones en el fin de año. [...] Ahora puedo soñar, ir atrás de esas cosas para darles ese placer a ellos (señalando los hijos) (mujer entrevistada). 
En relación al PAC las palabras más comunes de los beneficiarios que participaron de la entrevista fueron:

[...] cambió bastante.... Hasta amigos no tenía más, no voy en bares, restaurantes, ningún lugar, entonces las cosas estaban difíciles, pues no conversaba con nadie. Ahora tengo mis compañeros de trabajo para conversar. Además de tener el trabajo que permite mentalizar otro tipo de vida, más allá de la cesta del PAC, ganamos también un dinero [...] (varón entrevistado).

Una mujer respondió:

“[...] cambió completamente la relación con mi familia, con mi hijo que tiene 13 años, antes yo tenía problemas con el Consejo Tutelar. [...] El PAC me ayudó a entender que tengo que colocar mi hijo en la guardería y que el mayor vaya para la escuela, ¿entiende?" (mujer entrevistada).

En los relatos mencionados, se percibe que cuando las políticas públicas objetivan el nivel más elemental de seguridad protectora (alimentación, trabajo/renta, vivienda, salud, educación) el impacto es inmediato y de grandes proporciones en el contexto y en la vida cotidiana de las personas. En este sentido, reforzando el pensamiento de Sen, no es posible considerar el desarrollo y la justicia sin atender las necesidades elementares de la vida.

La constitución de la libertad sustantiva, a través de la ampliación de las libertades instrumentales demuestran que las referidas políticas contribuyen significativamente. Una de las entrevistadas del Canaán mencionó que la posibilidad de conquistar la vivienda propia cambió, inclusive, su relación con el trabajo:

"Después de que entré en el proyecto [..] conseguí dos promociones. Porque antes yo no conseguía [...] dar un algo más, porque estaba con inseguridad dentro de mí, pensaba en la inestabilidad de mis hijos. Después que vi mi casa [...], siendo construida, comencé a tranquilizarme porque sabía que mis hijos y yo tendríamos un lugar para vivir" (mujer entrevistada).

Otra entrevistada vinculó los efectos de la política con la posibilidad de contribuir para un futuro más promisorio para sus hijos/as:

"El dinero que está sobrando, lo estamos ahorrando para el piá *(sic) ${ }^{4}$, quiero que él estudie, que haga una facultad. Quiero que mi hijo tenga el estudio que el padre y yo no tuvimos" (mujer entrevistada).

La condición de agente activo para Sen representa la capacidad de tornarse sujeto de su vida, esta condición que está estrechamente conectada con las capacidades y la

\footnotetext{
${ }^{4}$ Piá es una jerga de Rio Grande do Sul, Brasil, para designar "hijo", "adolescente”.
} 
ampliación de la libertad posibilita diferentes elecciones, incluso sentirse responsable por los destinos de la propia vida. Al tratar este tema, una participante del proyecto Canaán relató un interesante dialogo con la representante del Grupo de Mujeres Unidos Venceremos:

Mi casa no está terminada, dame la chave que yo la voy a terminar. Ella me pregunto, ¿vos la conseguís terminar? Yo dije sí, yo trabajo y mi marido trabaja, quiero terminar mi casa. Quiero tener el orgullo de decir: yo coloqué tal cosa en mi casa, yo hice tal cosa para mi casa... también quiero hacer mi parte, mi esfuerzo (mujer entrevistada).

En la misma línea, se refirió un beneficiario del PAC:

Yo tenía otro problema, sofría de cataratas, casi no veía nada, la coordinadora podría haberme despedido, pues no veía más nada casi. Ella me encaminó para hacer la cirugía, y hoy veo bien con los dos ojos (varón entrevistado).

Otra beneficiaria del Canaán relata que antes no podía dejar los hijos jugar con los vecinos, pues no conocía quien vivía en la misma calle, ya que tenía que mudarse mucho porque no conseguía pagar el alquiler. Ahora conoce todo el vecindario y puede dejar los hijos jugar libremente en la calle. Con esto, se nota que el PAC también permitió construir espacios de relacionamiento de calidad entre sus beneficiarios, posibilitando entornos positivos de conversación, donde se desplieguen redes sociales de ciudadanía.

\section{Consideraciones finales}

Las políticas públicas son la identidad y misión de un Estado democrático. Esta convicción precisa integrar el imaginario de la población, especialmente de sus líderes más importantes, tanto de los gobiernos, cuanto de las demás organizaciones sociales, inversionistas e instituciones que, en forma conjunta y organizada, son responsables por la arquitectura organizacional de las sociedades y por las condiciones de justicia social de la población.

El análisis de programas sociales como el Proyecto Canaán y el Apoyar y comprometer, demuestran -desde el punto de vista simbólico y representativo- las condiciones para que las personas desenvuelvan su identidad personal, el ejercicio de la libertad, la ampliación de las capacidades (capabilities) y la participación social en sus diferentes estrategias, entre otras prerrogativas. Afirmamos que, comprender la contribución de Sen sobre justicia social y, específicamente, sobre políticas públicas es una referencia central para la estructuración de las condiciones de políticas públicas actualizadas que visen el bien común por medio de la superación de las desigualdades sociales escandalosas. Conocer aquellas políticas públicas puede contribuir en la tarea de corregir las posibles 
limitaciones, así como también para organizar proyectos actualizados a los contextos locales o de alcance más amplio; fomentando el desarrollo humano, social económico y sostenible sin depender -exclusivamente- del crecimiento económico.

\section{Referencias}

Fundação Pinheiro. (2017). Déficit habitacional en Brasil 2015: resultados preliminares. Belo Horizonte: FJP.

Kujawa, H. y Zambam, N. (2018). Conquista de la vivienda en el Loteamento Canaán en Passo Fundo, Brasil. MERCATOR, 17, e17031, Fortaleza.

Maricato, E. (2014). El impase de la política urbana en Brasil (3 ed.). Petrópolis, Rio de Janeiro: Vozes.

Nussbaum, M. C. (2015). Sin fines de lucro: Por qué la democracia necesita las humanidades. Traducido del inglés por Fernando Santos. São Paulo: Martins Fontes.

Rolnik, R. (2015). Guerra de los lugares: la colonización de la tierra y de la vivienda en la era de las finanzas. São Paulo: Boitempo.

Sen, A. (1999). Sobre ética y economía: Traducción de Laura Teixeira Motta. São Paulo: Companhia das letras.

Sen, A. (2000). Desarrollo como libertad. Traducción de Laura Teixeira Motta. São Paulo: Companhia das Letras.

Sen, A. (2001). Desigualdad reexaminada. Tradución de Ricardo Doninelli Mendes. Rio de Janeiro: Record.

Sen, A. (2011). La idea de justicia. Traducción de Denise Bottmann y Ricardo Doninelli Mendes. São Paulo: Companhia da Letras.

Sen, A. y Kliksberg, B. (2010). Las personas en primer lugar. Traducción de Bernardo Ajzemberg y Carlos Eduardo Lins da Silva. São Paulo: Companhia das letras.

Zambam, N. J. Kujawa, H. (2017). Las políticas públicas en Amartya Sen: condición de agente y libertad social/Public policies in Amartya Sen: agent condition and social freedom. Revista Brasileña de Derecho, 13(1), 60-85. https://doi.org/10.18256/2238-0604/revistadedireito.v13n1p60-85 